\title{
Richtlinien «Zusammenarbeit Ärzteschaft - Industrie»: definitive Fassung
}

\author{
Prof. Peter Suter, \\ Genf, Präsident SAMW
}

Prof. Werner Stauffacher, Basel, Leiter AG «Zusammenarbeit Ärzteschaft - Industrie»
Vor ziemlich genau einem Jahr veröffentlichte die Schweizerische Akademie der Medizinischen Wissenschaften (SAMW) eine erste Version der Richtlinien «Zusammenarbeit Ärzteschaft - Industrie» zur Vernehmlassung. Die Richtlinien sollten die 2002 erlassenen «Empfehlungen» zum gleichen Thema ablösen und waren, wie diese, von einer gemischten Arbeitsgruppe von Ärzten aus Klinik, Praxis, Forschung und Industrie, in der auch die FMH kompetent vertreten war, erarbeitet worden.

Die Richtlinien, wie die vorangegangenen Empfehlungen, galten dem Potential für Interessenkonflikte in Fortbildung und klinischer Forschung, denn angesichts des praktisch völligen Rückzugs der öffentlichen Hand, der Spitalträger und der Berufsorganisationen aus deren Finanzierung sind Interessenkonflikte beinahe unvermeidlich.

Die Reaktionen auf die Veröffentlichung waren teilweise vehement. Einerseits weil die Vernehmlassung seitens der SAMW schlecht kommuniziert, nicht als solche verstanden und die Publikation deshalb als Diktat empfunden wurde. Zum zweiten, weil der Text missverständliche Formulierungen enthielt, die einseitig die praktizierenden Kolleginnen und Kollegen zu exponieren und ihre Beteiligung an der klinischen Forschung in Frage zu stellen schienen; und zum dritten, weil die gegenüber den «Empfehlungen» verbindlicheren Bestimmungen der Richtlinien zu Fortbildungsveranstaltungen und Kongressen die Befürchtung weckten, die ärztliche Weiter- und Fortbildung werde erschwert und verteuert und damit gefährdet.

In der Folge beauftragte der Vorstand der SAMW die um einen Vertreter der interventionellen Disziplinen erweitere Arbeitsgruppe mit einer Überarbeitung der Richtlinien. Diese sollte einerseits den vorgebrachten Einwänden Rechnung tragen; andererseits sollte sie das Ziel der Richtlinien nicht verwässern, nämlich die Abhängigkeit der Ärzteschaft von privatwirtschaftlicher Finanzierung zu reduzieren, diese dort, wo sie unvermeidlich ist, bewusst und transparent zu machen, und das Bewusstsein und das «Sensorium» der Ärzteschaft für Interessenkonflikte zu schärfen.
Die revidierten Richtlinien liegen jetzt vor und sind im Internet abrufbar (www.samw.ch). Sie wurden am 24. November 2005 vom Senat der SAMW genehmigt und damit in Kraft gesetzt.

Änderungen betreffen die klinische Forschung (Kapitel I), wo primär die Vorschriften einfacher und besser verständlich formuliert wurden und die Praxis als Ort klinischer Forschung neu ausdrücklich erwähnt wird. Im Kapitel II (Aus-, Weiter- und Fortbildung) wurden die strengen Vorschriften bezüglich Monosponsoring und Kostenbeteiligung der Teilnehmer für kleine Veranstaltungen (z.B. lokaler Ärztevereine), spitalinterne regelmässige Veranstaltungen der Weiterund Fortbildung und - generell - für Ärztinnen und Ärzte in Weiterbildung etwas gelockert; im letzteren Fall allerdings nicht zugunsten von Sponsoring, sondern im Sinne der zumindest teilweisen Kostenübernahme durch den Arbeitgeber bzw. Kostenerlasses durch den Veranstalter. Bei der Annahme von geldwerten Geschenken und Naturalleistungen (Kapitel III) verweisen die Richtlinien nur mehr auf den einschlägigen Artikel der Standesordnung der FMH, betonen aber, dass dieser für Ärztinnen und Ärzte in Klinik, Praxis und Forschung gültig ist und insistieren auf der Unabdingbarkeit einer adäquaten Gegenleistung und eines verbindlichen Vertrags.

Der wohl wichtigste Satz der Richtlinien, den jede Ärztin und jeder Arzt dauernd vor Augen haben sollte, steht - im Sinn einer Maxime - ganz am Anfang des Texts und wurde sinngemäss (und in Englisch) vom American College of Physicians übernommen: «[...] wäre es mir recht, wenn mein Verhalten in meinem Umfeld bekannt würde?»

Vor einem Jahr wurden Universitäten, Spitäler, öffentliche Hand und Berufsverbände dazu aufgerufen, gemeinsam mit Ärztinnen und Ärzten dafür zu sorgen, dass die Zusammenarbeit zwischen Ärzteschaft und Industrie in Fortbildung und klinischer Forschung wieder von der Abhängigkeit zur Partnerschaft mutieren kann. Der Aufruf ist Anfang 2006 und für die neue Richtlinie noch immer gültig und schliesst auch die FMH mit ein. 